\title{
The Tapley and Bryden test of performance differences between the hands: The original data, newer data, and the relation to pegboard and other tasks
}

\author{
I C McManus \\ Research Department of Clinical, Educational and Health Psychology \\ University College London \\ Gower Street \\ London WC1E 6BT, UK \\ i.mcmanus@ucl.ac.uk \\ John Darrell Van Horn \\ USC Mark and Mary Stevens Neuroimaging and Informatics Institute \\ Keck School of Medicine of USC \\ University of Southern California \\ 2001 North Soto Street - Room SSB-102 \\ Los Angeles, CA 90032 \\ jvanhorn@usc.edu \\ Pamela J Bryden \\ Department of Kinesiology and Physical Education \\ 75 University Avenue West \\ Waterloo, Ontario, Canada, N2L 3C5 \\ pbryden@wlu.ca
}




\section{Summary}

Tapley and Bryden (T\&B)'s 1985 circle-marking task is a group-administered task assessing performance differences between the hands. The bimodal distribution clearly separates selfdescribed right- and left-handers. Using Phil's original datafiles we analyse the test in more detail, providing raw scores for each hands which are useful forensically, and we provide reliability estimates.

Van Horn's unpublished $1992 \mathrm{PhD}$ thesis studied T\&B tasks and Annett pegboards varying in difficulty. A striking finding, that Phil Bryden called "the Van Horn problem", was that hand differences (R-L) were unrelated to task difficulty. That result was the starting point for Pamela Bryden's 1998 thesis, firstly replicating Van Horn, but then showing task difficulty did relate to hand differences for grooved pegboards. Pamela Bryden's model for those effects is presented here.

Comparing across tasks, the T\&B and pegboard tasks showed almost complete consistency for direction of handedness. Likewise, within each task, degree of handedness intercorrelated strongly across variants. In strong contrast, degree of handedness for T\&B tasks showed minimal correlation with degree of handedness for pegboards. At the highest level, therefore, direction of handedness is consistent within individuals (conventional right and left handedness), but there are separable processes determining dominant-non-dominant hands differences for each particular task. 


\section{Introduction}

In 1985 Phil Bryden and his collaborator Marion Tapley ${ }^{1}$ published in Neuropsychologia a simple but effective test of performance differences between the right and left hands which was notable for being suitable for group administration (Tapley \& Bryden, 1985). They argued that a group test of performance rather than preference was required as the J-shaped distribution which resulted from handedness questionnaires was unlikely to reflect the underlying continuous distribution of handedness scores. The most popular test at that time for measuring handedness was the Annett pegboard developed by Marian Annett (Annett, 1972; Annett, 1976), which as Tapley and Bryden (T\&B) said, "requires equipment and is not readily administered to groups of subjects" (p.215) (Tapley \& Bryden, 1985).

The original T\&B test is shown in figure 1, participants being asked to "make a dot in each circle following the pattern as quickly as you can". The response sheet was produced using a typewriter and so where it says, "Mark a dot in each circle...", the circle is actually an ellipse formed by a capital "O", the exact size of which will be considered later. Participants were allowed 20 seconds on each trial, two trials of which used the writing hand and two used the non-writing hand, in counterbalanced order. The T\&B test was derived from the Test of Motor Impairment of Stott et al (Stott, Moyes, \& Henderson, 1972), which in turn was based on the Oseretsky Test of Motor Proficiency, originally published in Russian in 1923, and which became the Bruininks-Oseretsky Test of Motor Proficiency (Bruininks \& Bruininks, 2005). Close relatives can also be found in the tests of Borod et al (1984) and Steingrueber (1975).

The T\&B task was administered to 1556 students who were taking part in an introductory psychology course at the University of Waterloo, of whom 161 were self-described left-handers and the remainder were right-handers. A Laterality Index (LI) was calculated as $(R-L) /(R+L)$, where $R$ and $L$ are the number of circles marked with the right and left hands. The original paper shows a clearly bimodal distribution, with right-handers and left-handers having almost no overlap in their distributions. That bimodality was of much theoretical importance, as Marion Annett had argued strongly that the distribution of scores was unimodal with a 'right shift' (Annett, 1972; Annett \& Kilshaw, 1983; Kilshaw \& Annett, 1983). At much the same time as T\&B were publishing their data showing a bimodal distribution, one of us (ICM) had published a paper suggesting that bimodality was also visible in data collected by the National Child Development Study (NCDS) on a very large cohort study of UK children (McManus, 1985b) (Annett, 1985b; McManus, 1985a). In addition an analysis of data on the Annett pegboard using a method of decomposition of Gaussian mixtures suggested the presence of two separate distributions, although the overall distribution appeared unimodal because the difference in means was small compared with the standard deviations and hence the minor distribution had slipped into the tail of the major distribution.

Chris McManus first met Phil Bryden in 1987 and visited Waterloo many times before Phil's death in 1996, and a frequent topic of discussion was the nature of handedness, and the relation between preference asymmetries and performance asymmetries. One outcome of those discussions was that in 1989 Jack Van Horn began a PhD with McManus, a component of which was a detailed study of hand performance in a range of pegboard, circle marking, and other tasks (Van Horn, 1992). The handedness results in that study have not previously been published although they illuminate a number of key aspects of differences in performance between the hands. In 1994 Phil had received an early draft of a never published paper on Jack's thesis (Van Horn \& McManus, 1994), and in

\footnotetext{
${ }^{1}$ S. Marion Tapley Chiduck died in December 1998 aged 48, less than two years after Phil.
} 
various emails to McManus in the early part of 1996 he referred to "the Van Horn problem". The Van Horn results also influenced Pam Bryden, Phil Bryden's daughter, who studied the question further in her own PhD thesis supervised by Fran Allard and Colin Ellard in Waterloo ([Pamela] Bryden, 1998). On its first page, Pam Bryden's thesis said that the findings of Van Horn, "contradict the current theories accounting for manual asymmetries", which invoked differences in visual feedback (Flowers, 1975) or in controlling motor output ([John] Annett, [Marian] Annett, Hudson, \& Turner, 1979). Bryden's thesis began by replicating the findings of Van Horn, and then extending them in important ways, as we will describe below.

Here we will concentrate firstly on a more detailed analysis of the T\&B task which was possible as after Phil's death copies of the files on Phil's computers were provided to Chris McManus, with the assistance of Phil's wife, Pat Rowe. In particular we will describe in more detail the original T\&B test, giving data for the right and left hands separately. The latter are relevant to an issue raised by T\&B paper as to whether a Laterality Index or a simple difference score is a better measure of asymmetry. We will then consider the results of Van Horn, which were not previously available digitally, and therefore key chapters of the thesis have now been put online at http://www.ucl.ac.uk/medical-education/resources/Tests/VanHornAndMcManus-1994-DraftForWebsite.pdf. In a separate paper in this special collection, Pamela Bryden also provides a review of related recent literature ([Pamela] Bryden (2015)), although here we consider all of the key studies which look directly at the tasks used by Tapley and Bryden and by Van Horn.

Van Horn's data study used multiple variants of both the T\&B circle-marking and the Annett pegboard task in the same large group of right and left-handed participants. These data also illuminate the question of the best way of describing performance asymmetries, and in addition allow a calculation of the reliability of the various measures, separately for asymmetries within handedness groups, between right and left-handers, and for a binary measure of 'right preference' versus 'left preference'. We will also discuss an important paper by Marion Annett, published in 1992 (Annett, 1992), at the same time as Van Horn was submitting his PhD thesis. Finally we will relate the Van Horn data to the studies described in Pam Bryden's thesis, which in particular looked in detail at variants of the pegboard task.

Although the data described in this report are two or more decades old, the issues raised by them are still germane to modern research, both in theoretical and practical terms. The Annett pegboard in particular has been used in some large-scale cohort studies as the primary measure of handedness in a search for genes linked to handedness, so that understanding the measure better is important (Brandler et al., 2013; Francks et al., 2002; Scerri et al., 2011). Recently one of us (ICM) has also used the T\&B and Van Horn circle-marking tasks in a forensic context, which will be mentioned in the discussion, and the normative data here were useful in that context. For forensic use it is helpful to be able to describe the reliability of a test. Calculating the reliability of measures of laterality is not straightforward since the distributions are mostly bimodal or mixtures, and there is a need to distinguish reliability between-handedness groups and within-handedness groups and as a binary measure (McManus, 1983). We therefore also provide several measures of reliability for the Annett pegboard and the T\&B task.

\section{Method}

Two separate sets of raw data have been re-analysed.

$T \& B$ data. Tapley and Bryden collected data from students participating in eight separate classes over a period of two terms (Tapley \& Bryden, 1985), and the SPSS data file makes clear that this was 
in autumn 1979 and spring 1980. Details of the method are given in the T\&B paper, and need only to be reported here in brief. Figure 1 shows a version of the test used in Phil Bryden's lab which allowed measurement of the actual sizes of the response circles. The letter $\mathrm{O}$ had an external size of about $2.5 \mathrm{mms} \times 2.1 \mathrm{mms}$ and an internal size of about $1.8 \mathrm{mms} \times 0.8 \mathrm{mms}$. The line of the ' $\mathrm{O}$ ' is quite thick, and the target is equivalent to a circle with an external diameter of $2.3 \mathrm{mms}$ and internal diameter of $1.2 \mathrm{mms}$. The Os were about $45 \mathrm{mms}$ apart. Students were allowed 20 seconds to complete each part of the test. As well as data on the T\&B task, information was also available on age, sex, and self-reported handedness. A hand preference inventory was also administered, which can loosely be described as a 'modified Edinburgh Handedness Inventory' (mEHI) (Edlin et al., 2015), having the first seven items and then the ninth of the original Edinburgh Handedness Inventory (Oldfield, 1971) but with each item scored on a 5-item scale ([Phil] Bryden, 1977). The scores on the $\mathrm{mEHI}$ were converted to a standard laterality index ( $\mathrm{mEHI}-\mathrm{LI})$ in which the scores ranged from -100 for complete left-handers to +100 for complete right-handers.

Van Horn data. A group of 112 participants, balanced for sex and handedness (based on writing hand use in the McManus (1979) handedness questionnaire) was tested on variants of the Annett pegboard. A balanced group of 56 of these participants was also tested on variants of the T\&B task. Several other tasks, including the Bishop square-tracing task (Bishop, 1980), were also used, but are not described further here, but results can be found in the thesis (Van Horn, 1992), and are referred to in the form VH.165, indicating the page number in the thesis. For technical reasons, full raw data were not available for all of the tasks used in the thesis, but the analyses here are based on raw data, which have only recently been retrieved with some difficulty from obsolete computer tapes.

1. Circle-marking task. The variants of the T\&B circle-marking task were graded in difficulty using Fitt's Law (Fitts, 1954) with different circle diameters. The distance between circles was always set at $15 \mathrm{mms}$, with circles of diameter 10, 5.5, 3.3 and $2.5 \mathrm{mms}$ (for examples see the thesis (Van Horn, 1992)). A felt-tipped pen with a diameter of 2 mms was used for marking the circles. Fitts' Index of difficulty can be calculated as:

$$
I_{d}=-\log _{2} \frac{W}{2 \cdot A}
$$

where $A$ is the amplitude of the movement, and $W$ is the width of the target, calculated as Fitts suggested, as the difference between the size of the hole and size of the pen. The four tasks therefore had difficulties of 1.91, 2.10, 4.53 and 5.91 bits/response. Fitts' Index for the T\&B task, assuming the dimensions given earlier, and a typical pen or ballpoint diameter of half a millimetre, is $3.38 \mathrm{bits} /$ response. As in the T\&B study, 20 seconds were allowed for each trial. Statistical analysis used mixed effects analysis of variance, with handedness being between subjects, and hand used and circle diameter being within subjects.

2. The pegboard experiments. As well as the 'standard' Annett pegboard (Annett, 1970a; Annett, 1985a), Van Horn also followed John Annett et al (1979) in varying the pegboard dimensions (and an early unimanual experiment had developed that approach ([John] Annett, Golby, \& Kay, 1958). The dimensions of the pegboard are given in detail in the thesis, along with photographs. The two experiments used different pegboards, with the following variations:

a. Experiment 1. This used a 'universal pegboard' where the rows of pegs could be three different distances apart (DA1: 203, DA2: 305 or DA3: $406 \mathrm{mms}$ ), with four different hole spacings within a row (HS1: 15.0, HS2: 22.5, HS3: 30.0 or H4: 37.5 
$\mathrm{cms})^{2}$. Pegs were standard, with length $50 \mathrm{mms}$, and diameter $9.5 \mathrm{mms}$. The distances apart were based on the study of John Annett et al (1979). Statistical analysis used mixed effects analysis of variance, with handedness being between subjects, and hand used, peg distance and spacing being within subjects.

b. Experiment 2. A pegboard constructed to be as close as possible in dimensions to the standard Annett pegboard, with ten $12.7 \mathrm{mms}$ diameter holes, $37.5 \mathrm{mms}$ apart, and $203 \mathrm{mms}$ between the two rows of holes. The original specification uses pegs of length $50 \mathrm{mms}$ and diameter $9.5 \mathrm{mms}$ with rounded ends. We varied both the peg size and the peg ends. In contrast to John Annett et al (1979) Small (Sm) pegs were the standard size of $9.5 \mathrm{mms}$ diameter, whereas Large (Lg) pegs were $12.5 \mathrm{mms}$ diameter, which was a much tighter fit in the holes. The peg ends were either Rounded (Rd) as in the original experiment, or were Flat (Ft), being cut square, which requires more precision on insertion as the square cut ends can jam in the hole if the angle of entry is not correct, particularly with the large pegs. Statistical analysis used mixed effects analysis of variance, with handedness being between subjects, and hand used, peg size and peg end being within subjects.

Procedure. For experiment 2, as is conventional, each separate task was carried out four times, twice with each hand, either in the order $D, N D, N D, D$ or ND,D,D,ND, where $D$ is the dominant hand and ND is the non-dominant hand, the particular order being randomised within participants across conditions. Experiment 1 had more conditions and therefore each condition was carried out once with the dominant and once with the non-dominant hand, the order being randomised within subjects. A further difference in procedure from the standard Annett pegboard was that pegs were moved away from the participant, which probably makes the task a little harder as the holes in which the pegs are placed are further away and a little more oblique in outline. The Van Horn participants were also standing, a factor which has been shown also to increase movement times (Westwood, Roy, [Pamela] Bryden, [Phil] Bryden, \& Roy, 1998). Statistical analysis used mixed effects analysis of variance, with handedness being between subjects, and hand used and variations in pegs and distances being within subjects.

\section{Results}

The Tapley and Bryden task.

Overall the data file had information on 1559 participants, but a small number did not give information on some measures. $10.3 \%(161 / 1556)$ self-reported that they were left-handed. On the modified Edinburgh Handedness Inventory, scored as mEHI-LI , 8.7\% (131/1510) of participants had scores of 0 or less.

As is conventional in tests of performance, T\&B repeated their task four times, in the order D, ND, $N D$, and $D$. There was a small learning effect with a hand being faster on the second use than the first use for the dominant hand ( 52.0 vs 47.0 circles marked, paired t-test, $t=26.4,1543, p<.001$ ) and for the non-dominant hand ( 31.5 vs 29.1 , paired $t$-test, $t=18.8, p<0.001$ ), the proportional increase ((Time 2-Time1)/Time1) being slightly greater for the dominant hand (12.4\%) than the non-dominant hand (10.0\%) (paired t-test, $t=3.76,1543 \mathrm{df}, \mathrm{p}<.001)$. T\&B reported their results in terms of a conventional Laterality Index (T\&B-LI) calculated as $100 *(R-L) /(R+L)$, in which right-handers would be expected to have a positive T\&B-LI and left-handers a negative T\&B-LI. The T\&B-LI is plotted in

\footnotetext{
${ }^{2}$ Original measurements for the Annett pegboard were in inches, where the metric values described here make more intuitive sense, the pegs being $3 / 8^{\prime \prime}$ in diameter and placed in $1 / 2$ " diameter holes.
} 
figure 2, which shows very strong bimodality. T\&B did not give the distribution of circles marked by right and left hands, and it is shown in figure 3 . There is an almost perfect separation of selfreported right and left-handers, only $7(0.5 \%)$ right-handers performing better with the left hand, and $1(0.6 \%)$ left-handers performing better with the right hand (and these are similar proportions to those reported elsewhere (McManus, 1985b)).

Figure 4 shows the relationship between the modified Edinburgh Laterality Index (mEHI-LI) and the T\&B-LI, with self-reported handedness also indicated. A T\&B-LI of zero provides a good separation of self-reported right and left-handers, as is also the case with the $\mathrm{mEHI}-\mathrm{LI}$. The overall correlation between the $\mathrm{mEHI}-\mathrm{LI}$ and the T\&B-LI is large and highly significant $(r=.748, p<.001, n=1496)$, but within both right-handers and left-handers the correlation is much smaller although still significant (Right-handers, $r=.186, p<.001, n=1139$; left-handers, $r=.195, p=.014, n=157$ ).

Reliability of the T\&B-LI. Reliability is conventionally measured by the test-retest correlation, and that is relatively straightforward to do with the T\&B test. Performance of the right and left hands is each measured twice, so that two separate LIs can be calculated using R1 with L1 and R2 with L2. T\&B did that and reported a correlation of 0.81 (and we found a slightly higher correlation of 0.832 , the reasons for the difference not being entirely clear). The correlation between the measures on the two occasions is an indication of reliability, but it is however based on only half as much data as with the entire test. The correlation therefore needs to be corrected with the Spearman-Brown formula for a test of twice the length to obtain the reliability of the whole test (Ghiselli, Campbell, \& Zedeck, 1981). Table 1 shows the test-retest correlation of 0.832 and the estimated reliability of 0.908 .

The reliability calculated using the right and left hand scores is for the entire scale. However the distributions are bimodal and confound two separate types of reliability. How reliable is the T\&B test as a measure of the direction of handedness and how reliable is the T\&B test as a measure of the degree of handedness? Direction of handedness can be calculated as a positive or negative T\&B-LI, being -1 for left-handers and +1 for right-handers. The test-retest correlation for the binary scores is 0.932 , giving an estimated reliability of 0.965 . In contrast, degree of handedness (i.e. dominant hand in relation to non-dominant hand) asks how extreme is an individual's handedness (are they strongly handed or weakly handed)? Table 1 shows that the test-retest correlation is much lower, with a value of 0.551 , and hence an estimated reliability of 0.711 . Despite the T\&B test being a very good measure of direction of handedness, it is good, but not as good, as a measure of degree of handedness.

The Van Horn extensions of the T\&B tests. Van Horn used versions of the T\&B task with more precisely controlled circle sizes, varying in difficulty from 1.91 (easiest), 2.10, 4.53 to 5.91 (hardest) bits/response. Statistical analysis used a mixed model ANOVA, with Laterality Indices within handedness groups averaged 0.089 for the easiest task, through $0.101,0.132$ and 0.195 for the hardest task, the differences in laterality index being highly significant $(F(3,162)=24.6, p<0.001$; VH.165), with no differences between handedness groups $(F(3,162)=1.52, p=0.210)$. It may not seem surprising that the hardest task is the most lateralised, but a plot of the raw data, in figure 5 , showing the number of circles marked by the right and the left hands of right and left-handers at each difficulty level, shows a rather different picture It is now clear that the points lie parallel to the diagonal, being shifted above or below it by a fixed amount. The implication was that $R-L$ is constant across difficulty levels. That implication was confirmed in the ANOVA, where there was a highly significant effect of circle $(F(3,156)=693.2, p<0.001)$ and a difference between dominant and nondominant hands $(F(1,156)=318.9, p<0.001)$. The key result though was that the Hand used $x$ Circle 
interaction was not significant $(F(3,156)=0.93, p=0.426)$, confirming that the data in figure 5 are indeed parallel to the diagonal. The laterality index varied across conditions because $R+L$ was highest for the easiest task and lowest for the hardest task, but R-L was constant across tasks, so that a laterality index would be greater for the hardest tasks. The most surprising result, though, was that $\mathrm{R}-\mathrm{L}$ is constant across tasks.

The Van Horn variants of the pegboard tests

Figure 6 shows the mean time for the dominant and non-dominant hand in each of the sixteen variant pegboards. In experiment 1, ANOVA (VH.157-8) confirmed that times were longer when the rows of holes were further apart $(F(2,216)=1064.5, p<0.001)$ and the holes were spaced further apart within a row $(F(3,324)=278.8, p<0.001)$, although there was no interaction between the two effects $(F(6,648)=0.97, p=0.445)$. A key result though was that although there was a large difference between the dominant and non-dominant hands $(F(1,108)=126.4, p<0.001)$, there was no interaction between hand used and distance between the holes $(F(2,216)=1.27, p=0.284)$, spacing within rows $(F(3,324)=0.43, p=0.729)$, or the interaction of hand used, distance and spacing within rows $(F(6,648)=0.69, p=0.657)$. There were also no significant differences by handedness, or interactions of handedness and spacing. In experiment 2, ANOVA (VH.161-2) showed a large difference between dominant and non-dominant hands $(F(1,108)=107.8, p<0.001)$, and effects of peg size $(F(1,108)=913.1, p<0.001)$ and peg shape $(F(1,108)=1459.8, p<0.001)$, as well as their interaction $(F(1,108)=66.8, p<0.001)$. However, as with experiment 1 , there was no significant interaction of hand used with peg size $(F(1,108)=1.84, p=0.177)$, peg shape $(F(1,108)=3.15, p=0.079)$, or their interaction $(F(1,108)=0.55, p=0.460)$. There were also no significant differences of handedness with hand used, or with peg size or shape.

Taken overall it can be seen that the task took longer when the rows of pegs were further apart, the holes were more widely spaced within rows, the pegs were a tighter fit to the holes, and the pegs were square cut rather than rounded. However, in a result similar to that for the circle-marking task, the differences between the two hands were similar across conditions, the points for the dominant and non-dominant hands being parallel to one another in figure 6.

Reliability of the Annett pegboard. Raw data for each trial were available for experiment 2, of which the first condition was a standard Annett pegboard. Table 1 shows the test-retest correlations and the estimated reliability of the whole test (i.e. testing twice with the right hand and twice with the left hand). Reliability for the overall score $(L-R) /(L+R)$ is 0.757 , which is much lower than for the $T \& B$ task. Reliability within handedness groups (degree of handedness) was relatively lower at .616, and the reliability for identifying individuals as right or left-handed (direction of handedness) was lower still at 0.505 . The value of 0.757 for the overall test is comparable to the value of 0.69 reported for a group of subjects retested from seven weeks to eighteen months later ([Marian] Annett, Hudson, \& Turner, 1974).

The relationship between the T\&B task and the pegboard.

In the population the T\&B task clearly gave bimodal score distributions (e.g. figure 2), whereas studies using pegboards showed unimodal distributions skewed to the right. The latter cannot be seen so readily in the Van Horn data as the study had equal numbers of right-handers and lefthanders. The relationship of the T\&B task and the pegboard are theoretically important, and figure 7 summarises all of the data from the Van Horn study. The horizontal axis shows the averaged difference scores across all four T\&B tasks, whereas the vertical axis shows the averaged difference scores across all sixteen pegboard tasks. Right and left-handers are shown separately. Of the 56 
participants, all but seven are concordant in laterality for the T\&B and pegboard tasks. The T\&B task separates the right and left handers entirely, whereas the pegboard scores are not so good at doing that, four of the 28 right-handers and 2 of the 28 left-handers being in the 'wrong' half. The T\&B tasks show a very clear separation of the means of right and left-handers, which are about 4.8 standard deviations apart, whereas the pegboard tasks are only about 2.2 standard deviations apart, so that some right-handers appear to be better with the left hand at the pegboard task, and viceversa.

There is a correlation of 0.73 between the T\&B and pegboard scores in figure 7 (and disattenuating for the overall reliability of the scores gives a correlation of 0.88 , suggesting that pegboards and the $T \& B$ are measuring much the same thing across the population). However the correlation of $T \& B$ and pegboard scores within handedness groups (i.e. dominant hand vs non-dominant hand) is very small with a value of only 0.140 , which is non-significant.

\section{Discussion}

The Tapley and Bryden study is still one of the largest studies of a group-administered performance test of hand asymmetry, and the test, and variants of it, have proved useful in various research contexts. The original paper gave only a relatively crude population histogram, and in figure 2 we have provided a much more detailed version which shows the bimodality very clearly. The original paper also did not provide a scattergram of right-hand performance versus left-hand performance, with self-reported handedness indicated. Our figure 3 shows a clear bivariate bimodality, with right and left-handers almost perfectly separated.

One of us (ICM) was recently involved in a serious forensic case where the handedness of the suspect was a crucial part of the case. The data in figure 3 were of particular use as overall speed with each hand could be compared with population estimates. In principle it would be possible to 'fake left' or 'fake right' by performing particularly badly with what is actually the dominant hand. However by plotting right and left hand performances on figure 3 it was possible to exclude that option having occurred.

The relationship between the T\&B task, self-reported handedness, and the modified Edinburgh Laterality Inventory are explored in Figure 4. The T\&B-LI has the same direction as self-reported handedness in all but $0.6 \%$ of cases $(8 / 1544)$, whereas the $\mathrm{mEHI}$-LI has the same direction as selfreported handedness in all but $1.1 \%$ of cases $(16 / 1496)$. The modified Edinburgh Inventory is therefore slightly less good at corresponding to self-reported handedness.

The data from the study by Van Horn allow a much more detailed study of the process of the T\&B task. The Van Horn study used a series of four carefully-graded variants of the T\&B task, in which the difficulty was calculated using the method of Fitts (1992). An original version of the T\&B task used in Phil Bryden's lab also allowed an estimate of the Fitts difficulty for the original T\&B task.

A striking feature of the data for the four versions of the T\&B task used by Van Horn, shown in figure 5 , is that despite increasing difficulty of the versions of the test (and the overall speed was almost exactly proportional to the Fitts difficulties), the absolute difference in terms of circles marked by the dominant and non-dominant hands was effectively constant, the data in figure 5 being parallel with the diagonal, across and within task difficulty.

The analyses of the Van Horn data on pegboards of differing difficulty also show a similar result of a fixed right-left difference (see figure 6). For some comparisons, as between the different separations 


\section{Laterality: Asymmetries of Body, Brain and Cognition}

between the rows of pegs, and the different distances between the pegs in a row, it might be argued that the differences only reflect a relatively unskilled transport time, and if hand difference is primarily to do with fast, fine, distal movements of the hands, then little change in difference scores would be expected. However the final four columns of figure 6 show conditions with identical transport processes. The only variation is in the difficulty of putting the pegs into the holes due to size differences and some pegs being square cut, but while those manipulations clearly increase total time, they do not affect hand differences. The latter result is equivalent to the difference between the four T\&B tasks used by Van Horn, where again transport distances are identical and the only differences are with the final making of the pen mark within the circle.

Various other studies in the literature have mostly reported a fixed difference across conditions between right and left hands, despite other variation in task performance. In a developmental study, Marian Annett (1970b) showed a fixed R-L difference on the pegboard from the age of 6 to 15, despite overall times decreasing by about a third. Peters found in a single subject (Peters, 1976) and then in a series of 14 subjects (Peters, 1981) that while daily practice at ten trials per day for a median of 50 days increased speed at a tapping task, the difference between the hands showed no difference. A similar result was found with the Annett pegboard repeated daily for 26 days, speed overall increasing but the right-left difference remaining unchanged in two of the three participants ([Marian] Annett et al., 1974). However a thirty-day study on the pegboard in a single right-handed subject showed improvement in both hands, but with a greater rate of improvement in the right hand ([Pamela] Bryden \& Allard, 1998). Finally, the study of Flowers (1975) suggested that differences between the hands remain constant. The picture is, therefore, a little confused.

The only study before that of Van Horn which compared variants of the Annett pegboard seems to be that of John Annett et al (1979), the study having a complex design. The main experiment, which had three groups of 12 right-handed subjects, compared different separations of the rows of holes ('amplitude': 203, 305 and $406 \mathrm{mms}$ ) in a within subject design, and different hole sizes ('tolerance': $12.7,19.1$ and $25.4 \mathrm{mms}$ with $9.5 \mathrm{~mm}$ pegs) in a between subject design. A subsidiary study of twelve subjects used a single separation of $203 \mathrm{mms}$, but with all three hole sizes (12.7, 19.1 and $25.4 \mathrm{mms}$ with $9.5 \mathrm{~mm}$ pegs) in a within subject design. In the main experiment, there was no rightleft difference in relation to row separation ( $p=.26)$, but the right-left differences in relation to hole size effect was just significant ( $p=.046)$. In the subsidiary experiment, hole size had a highly significant effect on right-left differences $(p<0.001)$, and a post hoc analysis of just the standard separation in the main study suggested that the effect of hole size was also significant there $(p=.017)$. In general though the differences between the right and left hands were not influenced by row separation or hole size, despite these having highly significant effects on overall time, and in that sense the results are similar to the Van Horn study. The only exception is for the subsidiary experiment which is based on only 12 participants, but is highly significant. It differs from the Van Horn study in that: a) peg size is kept constant but hole size increased (and in the easiest condition there is a $9.5 \mathrm{~mm}$ peg in a $25.4 \mathrm{~mm}$ diameter hole, which is extremely easy, whereas the Van Horn study kept hole size constant and in the harder condition had a $12.5 \mathrm{~mm}$ peg in a $12.7 \mathrm{~mm}$ hole, which is very tight, a tolerance possible because the Van Horn apparatus used milled pegs made from a hard red plastic called Delron which were placed into brass holes); b) in the John Annett study the participants moved the pegs towards themselves, whereas in the Van Horn study the participants moved the pegs away from themselves; and c) in the Annett study, the participants carried out each single trial of moving ten pegs five times instead of the conventional two (i.e. RLRLRLRLRL or LRLRLRLRLR instead of RLLR or LRRL). Comparing times in a 'standard' condition, the John Annett subjects took 8.46 and 9.72 seconds (diff $=1.26$ seconds) with the dominant and non- 
dominant hands, compared with 8.03 and 8.57 seconds (diff $=0.54$ seconds) in the Van Horn studies, so that the Van Horn subjects were apparently processing the stimuli more quickly, suggesting that motivational differences are not responsible for the lack of an interaction in the Van Horn studies. Given that the Van Horn study had 112 subjects compared with 48 subjects in the John Annett study, that each subject in the Van Horn study carried out a total of $(12 \times 2+4 \times 4)=40$ pegboards, compared with $3 \times 10=30$ pegboards in the Annett study, and that all comparisons in the Van Horn study were within-subject whereas some in the John Annett study were between-subject and hence had less power, it seems reasonable to conclude that the Van Horn study is an adequate replication of the John Annett study, and that it has to be concluded that dominant-nondominant hand differences do not change as the difficulty of pegboards alters. Subsequently the thesis by Pamela Bryden also used variations on the Annett pegboard (see below) ([Pamela] Bryden \& Roy, 1999).

Most studies of lateralisation use a laterality coefficient to take into account individual differences in overall performance level. Indeed, Tapley and Bryden comment that,

\footnotetext{
"To score this test the number of circles properly filled was counted for each trial. Hand differences were then expressed as the difference between right- and left-hand scores divided by the total $(R-L) /(R+L)$. This measure represents the difference in rate expressed as a proportion of the overall rate. It was chosen, in part, because of a feeling that the difference between 100 and 98 circles filled represented performance similar to that seen in a performance of 200 and 196, rather than 200 and 198." (p.216; our emphasis).
}

Tapley and Bryden were correct that a laterality index such as $(R-L) /(R+L)$ feels as if it ought to be better than a simple difference score, $(R-L)$, but the present data suggest that may not actually be the case, either for the T\&B task or the pegboards.

Tapley and Bryden, like others looking for Laterality Indices, wanted a measure of lateralisation which is independent of overall performance, and $(R-L) /(R+L)$ intuitively feels as if it ought to fit the bill. However a little consideration suggests that that intuition may be wrong. Figure 8 shows a diagram with right-hand performance, $R$, on the $\mathrm{X}$-axis and left-hand performance, $L$, on the $Y$-axis. The solid main diagonal therefore indicates equal performance of the two hands. The total performance of the $R$ and $L$ hands, $p$, is $p=R+L$. Rearranged that formula becomes $L=p-R$, which for any value $p$ provides an iso-performance line, which will be at right-angles to the main diagonal. Isoperformance lines in figure 8 are shown as dashed red lines, which cross the main diagonal at $(p / 2$, $p / 2)$. Consider now a laterality coefficient calculated as $i=(R-L) /(R+L)$. The formula can be rearranged to become $L=R .(1-i) /(1+i)$. For any particular value of $i$ it is the case that $(1-i) /(1+i)$ is a constant, and hence $L$ will be a linear function of $R$, of the form $L=k . R$, for which it is clear that when $R=0$ so $L$ must also be zero. For a particular laterality index, $i$, the locus of the 'iso-laterality line' must therefore go through the origin, $(0,0)$. Iso-laterality lines are shown in blue in figure $8 \mathrm{a}$, the lines for different laterality indices fanning out from the origin, where all of the lines intersect. Instead of a conventional laterality index, figure $8 \mathrm{~b}$ instead shows lateralisation as a simple difference score, calculated as $d=R-L$. Re-arranging gives $L=R-d$, so that the locus of the points for any particular difference score, the iso-difference lines, will be one of the blue lines running parallel to the main diagonal, but shifted up by a factor of $R-L$.

If lines in a graph are uncorrelated with one another and hence are independent, then they will be at right angles to one another (and if the lines are not orthogonal then the cosine of the angle between the lines will be the correlation between them). However in figure $8 a$ it can be seen that the isolaterality lines and the iso-performance lines are not perpendicular to one another, but intersect at angles other than right angles, and therefore will be correlated. If the intention of using $(R-L) /(R+L)$ is 
to obtain a laterality index which is independent of $(R+L)$ then that intention must fail. More problematic is that the angle between the iso-laterality lines and the iso-performance lines will differ at different laterality indices, the angle of intersection varying as one moves along an isoperformance line. In passing it is also worth noting that the only lines in figure 8a which would always be orthogonal to the iso-laterality lines is the set of concentric circles based at the origin, but these take values of the form $\operatorname{sqrt}\left(R^{2}+L^{2}\right)$, and that is not a naturally obvious way to indicate overall task performance.

The iso-difference lines for the simple difference between right and left hand performance, $R-L$, are shown in figure $8 \mathrm{~b}$ by the blue lines, and it can be seen that these are always perpendicular to the iso-performance lines, and hence the difference scores as a laterality index is uncorrelated with level of performance. On theoretical grounds there may therefore be more to be said for a laterality index based on a simple difference score, $R-L$, than for an index such as $(R-L) /(R+L)$ which attempts to correct for overall performance.

An intriguing feature of figure $8 \mathrm{~b}$, which at first may seem wrong, is that for right-handers there is a point at which while the right-hand is still performing, the left-hand has reached zero performance. That may occur rarely for motor tasks such as pegboards or dot-filling, but for very highly skilled tasks it indeed occurs. The most obvious example is writing, for which the quality of writing deteriorates in both hands as task demands increase (small size, high speed, etc) and there reaches a point where dominant hand performance is still acceptable (the writing is legible), but non-dominant hand reaches a performance of zero, being utterly illegible, or there not even being a written product. That catastrophic failure by the non-dominant hand is the model of figure $8 \mathrm{~b}$ and not figure $8 a$, which would predict a more graceful and parallel degradation of performance by both hands.

The question of whether or not differences between hands are fixed, irrespective of task difficulty, is a key one, and has been looked at most thoroughly for pegboards in Pam Bryden's PhD thesis (Bryden, 1998), and a series of subsequent papers. The thesis begins in experiment 1, by replicating the Van Horn finding, and then in Experiment 2 obtaining a similar result using Fitts' reciprocal tapping task (Robinson, 1985) with different sizes of target and stamp. The first exception to the Van Horn result occurred using the Grooved Pegboard (Reitan \& Davison, 1974) (Thompson, Heaton, Matthews, \& Grant, 1987), in which each peg also has a portion sticking out like a key, which has to be engaged in the 'keyholes' whose orientation varies. Pam Bryden not only looked at the time taken to insert the pegs (which is slow and requires a lot of skill) but also to remove the pegs (which requires far less skill and is much easier). In the 'remove' phase there was only a small hand difference, whereas there was a large one in the 'place' phase ([Pamela] Bryden \& Roy, 1999; [Pamela] Bryden \& Roy, 2005). Experiments 4 and 5 confirmed the effect with a 'grooved' version of the Annett pegboard. The studies from Bryden's PhD thesis, as well as later results on the Grooved Pegboard ([Pamela] , Roy, Rohr, \& Egilo, 2007) therefore show that while for a range of task difficulties the hand difference is fixed, as tasks become yet more complex so hand differences then increase with task difficulty (and it could be argued that the most complex of the hand tasks in everyday life, writing, shows the end result of that progression very clearly, with the non-dominant hand hardly able to write at all).

In neuropsychological terms, it is not easy to see how laterality as a fixed constant might happen, as occurred in the Van Horn tasks. John Annett et al (1979) carried out a careful review of various theories of the differences between the hands. One of the theories cited is the influential theory of Crossman and Goodeve, originally presented at a meeting of the Experimental Psychology Society in Oxford in 1963. It remained unpublished, though, and circulated only in mimeographed form until 
(and we were gratified to find that our slowness in publication was not unique), the paper was eventually published in 1983, twenty years after the original report (Crossman \& Goodeve, 1983; Wing, 1983). John Annett and his colleagues carried out a detailed frame-by-frame analysis of film of four subjects moving pegs, which suggested to them that hand differences are not due to differences between the hands in the efficiency of processing feedback, and neither is it due to differences between ballistic and controlled movements. Eventually they concluded that "It seems therefore that differences between hands in the control of aiming movements are [likely to be due to] greater variability in the mechanism for initiating these movements" (p.651), adding in the abstract that the, "motor output of the non-preferred hand is simply more variable". It is an interesting theory, but it is not clear that it can explain all of the Van Horn data. In some of the tasks the transport distance from start to the target is the same (the T\&B tasks, as well as altering only peg size and shape in the pegboard tasks), and therefore a fixed aggregate distance in arrival location should be expected, which will take time to correct but will be similar in the dominant and nondominant hands. However for tasks where the transport distance varies (altering the distance between rows or between holes within rows) the greater initial variability should produce an increasing error with increasing distance, and that should take longer to correct for the nondominant hand as on average the initial location will be further from the target than for the dominant hand.

A model for interpreting the various results was put forward by Pam Bryden in her PhD thesis (Bryden, 1998) (pp.93-102) and is shown schematically in figure 9. Relatively unskilled tasks can be performed by either hand using the motor control centres in each hemisphere, which have a direct line to the contralateral hand. However the motor output controlling the left hand is inherently noisier, so that performance is less good. More complex movements involving complex timing sequences or visuomotor integration and feedback control however require the lateralized motor centre, which typically is in the left hemisphere. The lateralized motor centre has a direct link to the motor control centre for the right hand, but only an indirect link via the corpus callosum to the inherently noisier motor centre for the left hand. Such a model can explain the broad pattern of relatively constant differences between the hands for lower level skills, but an increasing advantage to the right hand as skills become ever more complex. The model also has implications for that other great component of handedness, which is hand preference as opposed to differences in hand skill. In a separate paper in this issue, Pam Bryden (2015) integrates a range of studies which examine how control moves from the dominant to the non-dominant hand or vice-versa, in relation both to task complexity, and the bio-mechanical constraints of working across the midline into the opposite hemispace. Hand preference and skill differences are therefore related, although not in a strict oneto-one relationship, but with the relationship depending on task and spatial factors. An interesting challenge for the future would be to test that model directly either within an fMRI scanner or with other scanning methods.

Although the literature contains various measures of performance differences between the hands, much rarer are studies which carry out multiple skill measures on the same participants. An exception is the study of Marion Annett (1992), which has large numbers of participants, although the data reported are relatively limited, mostly being univariate, and most participants carried out only the pegboard and one of the four other tests. Correlations of (R-L)\% with pegboard performance are reported and average 0.51 (range 0.38 to 0.65 ). These are a little lower than the test-retest correlation of 0.609 reported in our table 1, but are compatible. The 1992 paper is also important for its discussion of unimodality and bimodality, and the study begins by saying a priori that one of the main features looked for in a group test to replace the pegboard, "was a unimodal 


\section{Laterality: Asymmetries of Body, Brain and Cognition}

normal curve" (p.583). However two of the tests (DOTS and HOLES) "were clearly bimodal" (p.596) and their distributions were similar to that of the T\&B test. Marian Annett then asks rhetorically, "Does the finding of bimodal distributions of R-L\% invalidate the RS theory as suggested by McManus (1985)?". However she then continues that "It is unlikely that the RS theory would have been formulated without the strong evidence for continuous and unimodal distributions... The theory now rests on considerably more evidence than could be invalidated by the discovery of bimodality for some tasks...". The section concludes that, "No one type of task has a monopoly as the measure of hand skill. The fact that tasks differ for extent of overlap between right and left handers raises interesting questions as to the nature of the skills involved" (p.596). The latter is indeed correct and will be discussed below. As to the issues of unimodality and bimodality, the reader is left to make up their own mind on those questions.

The Van Horn study was (and still is) very unusual in using multiple variations of several different tests, including the T\&B and pegboard tests, as shown in figure 6 in particular, which has a number of important implications. Taking the R-L scores overall it is clear that there is a strong disattenuated correlation of 0.88 between scores on the T\&B tasks and the pegboards. Laterality scores must always however be treated with statistical care as distributions are often bimodal and hence are mixture distributions (McManus, 1983), and correlations are particularly vulnerable to being misinterpreted. The overall high correlation in figure 7 between R-L differences on the pegboard and the T\&B task mainly reflects the fact that participants are almost entirely in the top right and bottom left quadrants. In other words, their direction of lateralisation on the two tasks is almost always the same (although in figure 7 there is a handful of cases who seem to have crossed lateralisation, and may be worth investigating further, and in terms of the model in figure 9 they would be difficult to explain). Despite the overall strong correlation, looking within, say, only the top right quadrant or only the bottom left correlation it is far from obvious that there is any correlation at all between R-L scores on the two different tasks. Calculating D-ND, a measure of the relative superiority of the dominant over the non-dominant hand, irrespective of handedness, shows that the correlation is actually 0.140 , which effectively is zero. That is a surprising and important finding. If a person is right-handed for task $A$ then they are very likely to be right-handed for task $B$, but if their dominant hand is very much better than their non-dominant hand for task $A$ then that has no predictive ability for how much better their dominant hand will be compared with their non-dominant hand on task $B$. Although we do not show the results here, we have also analysed the Bishop square tracking task and a computerised pursuit tracking task which are also in the Van Horn study. Factor analysis of right and left hand scores across all the methods finds a single main factor, which reflects right or left handedness. However factor analysis of dominant-nondominant difference scores across $a$ range of different types of measures finds separate factors for each method, with little evidence of a general factor, a result equivalent to that shown in figure 6 . In contrast, factor analysing the difference scores for, say, the 16 pegboard tasks carried out by the Van Horn subjects, gives a clear single factor (first eigenvalues of 5.75, 1.31, 1.09, 1.01, 0.94, etc). The average correlation between any two tasks is 0.31 (and that means that if all 16 pegboard scores are combined then the resulting score has a Cronbach's alpha of 0.878). A similar analysis for the four T\&B tasks of Van Horn gives an average inter-task correlation of 0.617 and a Cronbach's alpha of 0.857 . The scores for the individual participants shown in the separate quadrants of figure 7 therefore represent reliable differences between individuals within handedness groups. That has implications for the claim of John Annett et al (1979), quoted earlier, that "motor output of the non-preferred hand is simply more variable". It cannot be that simple as individuals appear to be different in the extent of their variability, at least within a type of task, but that variability seems to be independent across different motor tasks (such 
as circle-marking and using a pegboard). Once again, that has to have implications for the motor processes underlying hand preference.

In his thesis, Van Horn said, "it is unclear whether hand performance should be considered as uni- or multi-dimensional" (p.146).The answer would seem to be that differences between the right and left hands are unidimensional, with two clear classes of right and left handers (direction of handedness), whereas differences between the dominant and non-dominant hands (degree of handedness) are multidimensional, with the various factors being task specific.

Explaining the differences between tasks, as shown in figure 6, is far from straightforward. A useful starting point is the question at the end of the paper of Marion Annett (1992), where it is asked, "What skills have been measured?". Annett recognises that her various tasks require different skills, not least because they are of different magnitude, some requiring finger-thumb grasps, some needing impulsive or ballistic movements whereas others are continuous, and some involving proximal muscle usage, particularly for transport. It perhaps would not be surprising to find that there are only small D-ND correlations across tasks within individuals, since different skills are involved. R-L correlations though behave differentl. It is as if an additional fixed factor has been added to the dominant side which affects the right side in right-handers and the left-side in lefthanders. The present data show the clear problems of assuming that explanations of differences between right and left-handers can be extrapolated to explanations of differences between strong right-handers and weak right-handers, and vice-versa. Differences between right and left-handers and differences between strong and weak right-handers (or strong and weak left-handers) are due to different processes and different underlying mechanisms.

The present analyses, two decades after the death of Phil Bryden, and over three decades since the seminal Tapley and Bryden paper, show the importance both of the data in that original study and the subsequent experiments by Van Horn, and then by Pamela Bryden, which it helped to inspire. Those datasets, taken together, and to some extent influenced by the passage of time which allows different theoretical perspectives, show how little is still understood about the nature of handedness itself - that difference between the hands of which every right-hander and left-hander is entirely aware from their own behaviour, but for which we have almost no adequate scientific explanation. 
Table 1. Test-retest correlations and estimates of reliability for T\&B task and pegboard task used in their typical forms of a single use in the order RLLR or LRRL.

\begin{tabular}{|c|c|c|c|c|c|c|}
\hline & \multicolumn{2}{|c|}{$\begin{array}{c}\text { Regree of lateralisation } \\
\text { Reft Hand score } \\
\text { Lominant hand vs non- } \\
\text { Dominant hand) }\end{array}$} & \multicolumn{2}{|c|}{$\begin{array}{c}\text { Direction of } \\
\text { lateralisation } \\
\text { (Difference }>0 \text { or <0) }\end{array}$} \\
\hline $\begin{array}{c}\text { Test- } \\
\text { Retest }\end{array}$ & Reliability & $\begin{array}{c}\text { Test- } \\
\text { Retest }\end{array}$ & Reliability & $\begin{array}{c}\text { Test- } \\
\text { Retest }\end{array}$ & Reliability \\
\hline $\begin{array}{c}\text { T\&B } \\
\text { original data }\end{array}$ & 0.832 & $\mathbf{0 . 9 0 8}$ & 0.551 & $\mathbf{0 . 7 1 1}$ & 0.932 & $\mathbf{0 . 9 6 5}$ \\
\hline $\begin{array}{c}\text { Pegboard (Van Horn } \\
\text { Experiment 2, small , } \\
\text { round-ended pegs) }\end{array}$ & 0.609 & $\mathbf{0 . 7 5 7}$ & 0.445 & $\mathbf{0 . 6 1 6}$ & 0.338 & $\mathbf{0 . 5 0 5}$ \\
\hline
\end{tabular}




\section{Figure captions}

Figure 1. A version of the T\&B test used in Phil Bryden's laboratory at the University of Waterloo. Undated, but from the font it is probably later than the version in the 1985 paper.

Figure 2. Distribution of Laterality Indices in the T\&B data (T\&B-LI).

Figure 3. Numbers of circles marked with the right hand the left hand in the T\&B data. Open circles: self-reported right handers, Solid circles: self-reported left handers.

Figure 4. Laterality indices on the Tapley and Bryden task (T\&B-LI) in relation to modified Edinburgh handedness scores (mEHI-LI). Open circles: self-reported right handers, Solid circles: self-reported left handers. Solid lines indicate regression of T\&B-LI on mEHI-LI within handedness groups.

Figure 5. Numbers of circles marked with the right hand the left hand in the Van Horn data using variants of the T\&B task. Open symbols: self-reported right handers, Solid symbols: self-reported left handers. The different colours and shapes correspond to the hardest task (red inverted triangles), through blue upright triangles, green squares, and black circles for the easiest task.

Figure 6. Left axis: Mean time (secs) to move the ten pegs in the Van Horn study using sixteen variants of the pegboard. Solid circles: Time for the dominant hand ( $\pm 1 \mathrm{SE}$ ); Open circles: Time for the non-dominant hand ( $\pm 1 \mathrm{SE}$ ). Right axis: Differences in time for dominant vs non-dominant hand. The conditions are shown in the labels: Experiment 1: Distance apart (DA) and Hole Spacing (HS); Experiment 2: Small vs Large; Rounded end vs Flat end

Figure 7. Right and left hand differences for all tasks carried out in the Van Horn study. Horizontal axis: Average difference in number of circles marked in the four T\&B tasks. Vertical axis: Average difference in time for the sixteen pegboard tasks. Note that differences are scored so that righthanders score positively on both tasks. Open circles: right handers, Solid circles: left handers.

Figure 8. The relationship between measures of laterality (blue solid lines) and overall measures of performance (red dashed lines). a) shows lines of equal lateralisation for the conventional laterality index, $(R-L) /(R+L)$, and $b)$ shows lines of equal lateralisation for a simple difference score (R-). 
Reference List

Annett, J., Annett, M., Hudson, P. T. W., \& Turner, A. (1979). The control of movement in the preferred and non-preferred hands. Quarterly Journal of Experimental Psychology, 31, 641652.

Annett, J., Golby, C. W., \& Kay, H. (1958). The measurement of elements in an assembly task - the information output of the human motor system. Quarterly Journal of Experimental Psychology, 10, 1-11.

Annett, M. (1970a). A classification of hand preference by association analysis. British Journal of Psychology, 61, 303-321.

Annett, M. (1970b). The growth of manual preference and speed. British Journal of Psychology, 61, 545-558.

Annett, M. (1972). The distribution of manual asymmetry. British Journal of Psychology, 63, 343-358.

Annett, M. (1976). A coordination of hand preference and skill replicated. British Journal of Psychology, 67, 587-592.

Annett, M. (1985a). Left, right, hand and brain: the right shift theory. New Jersey: Lawrence Erlbaum.

Annett, M. (1985b). Which theory fails? A reply to McManus. British Journal of Psychology, 76, 1729.

Annett, M. (1992). Five tests of hand skill. Cortex, 28, 583-600.

Annett, M., Hudson, P. T. W., \& Turner, A. (1974). The reliability of differences between the hands in motor skill. Neuropsychologia, 12, 527-531. 
Annett, M., \& Kilshaw, D. (1983). Right and left-hand skill: Il. Estimating the parameters of the distribution of L-R differences in males and females. British Journal of Psychology, 74, 269283.

Bishop, D. V. M. (1980). Handedness, clumsiness, and cognitive ability. Developmental Medicine and Child Neurology, 22, 569-579.

Borod, J. C., Koff, E., \& Caron, H. S. (1984). The Target Test: A brief laterality measure of speed and accuracy. Percept.Mot.Skills, 58, 743-748.

Brandler, W. M., Morris, A. P., Evans, D. M., Scerri, T. S., Kemp, J. R., Timpson, N. J. et al. (2013). Common variants in left/right asymmetry genes and pathways are associated with relative hand skill. PLoS Genetics, 9(9), e1003751 ( doi:10.1371/journal.pgen.1003751).

Bruininks, R. H., \& Bruininks, B. D. (2005). BOT-2: Bruininks-Oseretsky test of motor proficiency (2nd edition). Minneapolis, MN: Pearson Assessments.

Bryden, M. P. (1977). Measuring handedness with questionnaires. Neuropsychologia, 15, 617-624.

Bryden, P. J. (1998). The origins of manual asymmetries: What is revealed by pushing the limits of task difficulty (PhD thesis). Waterloo, ON.: University of Waterloo (available at https://uwspace.uwaterloo.ca/handle/10012/301?show=full ).

Bryden, P. J. (2015). The influence of M.P.Bryden's work on lateralization of motor skill: Is the preferred hand selected for and better at tasks requiring a high degree of skill? Laterality, Submitted.

Bryden, P. J., \& Allard, F. (1998). Does Extended Practice Affect the Magnitude of Manual Asymmetries on a Pegboard Task? Brain and Cognition, 37(1), 44-46.

Bryden, P. J., \& Roy, E. A. (1999). Spatial task demands affect the extent of manual asymmetries. Laterality, 4(1), 27-37. 
Bryden, P. J., \& Roy, E. A. (2005). A new method of administering the Grooved Pegboard Test: Performance as a function of handedness and sex. Brain and Cognition, 58, 258-268.

Bryden, P. J., Roy, E. A., Rohr, L. E., \& Egilo, S. (2007). Task demands affect manual asymmetries in pegboard performance. Laterality, 12(4), 364-377.

Crossman, E. R. F. W., \& Goodeve, P. J. (1983). Feedback control of hand-movement and Fitts' Law [Paper presented at the meeting of the Experimental Psychology Society, Oxford, July 1963]. Quarterly Journal of Experimental Psychology, 35A(2), 251-278.

Edlin, J. M., Leppanen, M. L., Fain, R. J., Hackländer, R. P., Hanaver-Torrez, S. D., \& Lyle, K. B. (2015). On the use (and misuse?) of the Edinburgh Handedness Inventory. Brain and Cognition, 94, $44-51$.

Fitts, P. M. (1954). The information capacity of the human motor system in controlling the amplitude of movement. Journal of Experimental Psychology, 47, 381-391.

Flowers, K. (1975). Handedness and controlled movement. Br.J.Psychol., 66, 39-52.

Francks, C., Fisher, S. E., MacPhie, L., Richardson, A. J., Marlow, A. J., Stein, J. F. et al. (2002). A genomewide linkage screen for relative hand skill in sibling pairs. American Journal of Human Genetics, 70, 800-805.

Ghiselli, E. E., Campbell, J. P., \& Zedeck, S. (1981). Measurement theory for the behavioral sciences. San Francisco: W H Freeman.

Kilshaw, D., \& Annett, M. (1983). Right- and left-hand skill I: Effects of age, sex and hand preference showing superior skill in left-handers. British Journal of Psychology, 74, 253-268.

McManus, I. C. (1979). Determinants of laterality in man. University of Cambridge: Unpublished PhD thesis (available at http://www.ucl.ac.uk/medical-education/publications/phd).

McManus, I. C. (1983). The interpretation of laterality. Cortex, 19, 187-214. 
McManus, I. C. (1985a). On testing the right shift theory: a reply to Annett. British Journal of Psychology, 76, 31-34.

McManus, I. C. (1985b). Right- and left-hand skill: failure of the right shift model. British Journal of Psychology, 76, 1-16.

Oldfield, R. C. (1971). The assessment and analysis of handedness: the Edinburgh inventory. Neuropsychologia, 9, 97-113.

Peters, M. (1976). Unilateral control of bilaterally symmetrical movement as factor in lateralization of speech. Perceptual and Motor Skills, 42, 841-842.

Peters, M. (1981). Handedness: effect of prolonged practice on between hand performance differences. Neuropsychologia, 19, 587-590.

Reitan, R. M., \& Davison, L. A. (1974). Clinical Neuropsychology: Current status and applications. Washington, DC: Winston.

Robinson, R. G. (1985). Lateralized behavioral and neurochemical consequences of unilateral brain injury in rats. In S.D. Glick (Ed.), Cerebral lateralization in non-human species (pp. 135-156). Orlando: Academic Press.

Scerri, T. S., Brandler, W. M., Paracchini, S., Morris, A. P., Ring, S. M., Richardson, A. J. et al. (2011). PCSK6 is associated with handedness in indivuduals with dyslexia. Human Molecular Genetics, 20(3), 608-614.

Steingrueber, H. J. (1975). Handedness as a function of test complexity. Perceptual and Motor Skills, 40, 263-266.

Stott, D. H., Moyes, F. A., \& Henderson, S. E. (1972). Test of motor impairment. Guelph, Ontario: Brook Educational Publishing Limited. 
Tapley, S. M., \& Bryden, M. P. (1985). A group test for the assessment of performance between the hands. Neuropsychologia, 23, 215-221.

Thompson, L. L., Heaton, R. K., Matthews, C. G., \& Grant, I. (1987). Comparison of preferred and nonpreferred hand performance on four neuropsychological motor tasks. Clinical Neuropsychologist, 1(4), 324-334.

Van Horn, J. D. (1992). Brain structural abnormality and laterality in schizophrenia. University College London: Unpublished PhD thesis.

Van Horn, J. D., \& McManus, I. C. (1994). Task difficulty and hand differences in peg moving, circle marking and square tracing. Unpublished manuscript: Available at http://www.ucl.ac.uk/medicaleducation/resources/Tests/VanHornAndMcManusCircleMarkingTask.

Westwood, D., Roy, E. A., Bryden, P. J., Bryden, M. P., \& Roy, P. E. (1998). Increasing Postural Demands May Affect the Magnitude of manual Asymmetries. Brain and Cognition, 37(1), 4043.

Wing, A. M. (1983). Crossman and Goodeve (1963): Twenty years on. Quarterly Journal of Experimental Psychology, 35A(2), 245-249. 


\section{Prelerred Hand}

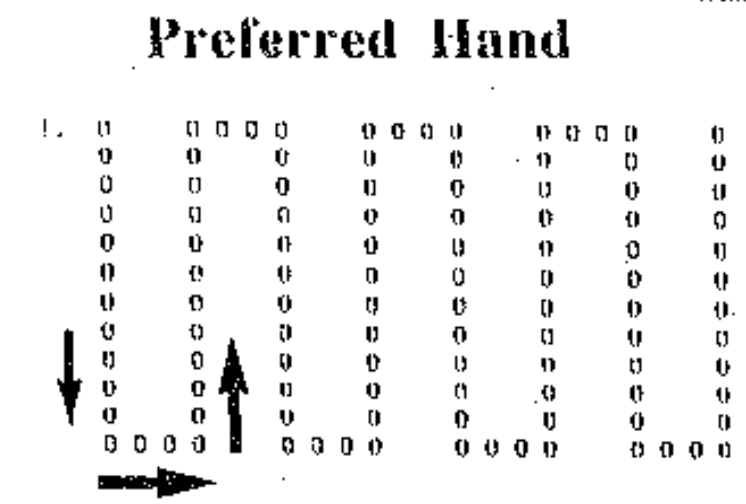

\section{Non-preferred Hand}

\section{Mark a fot in each circle following direction illobicated.}

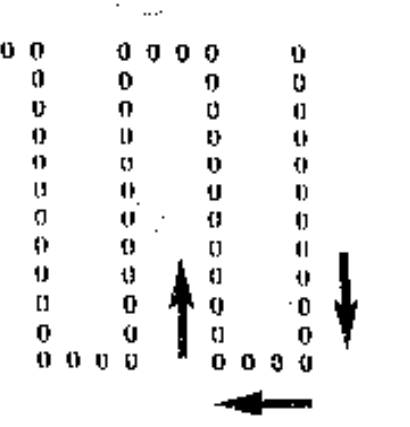

Non-preferred Hand

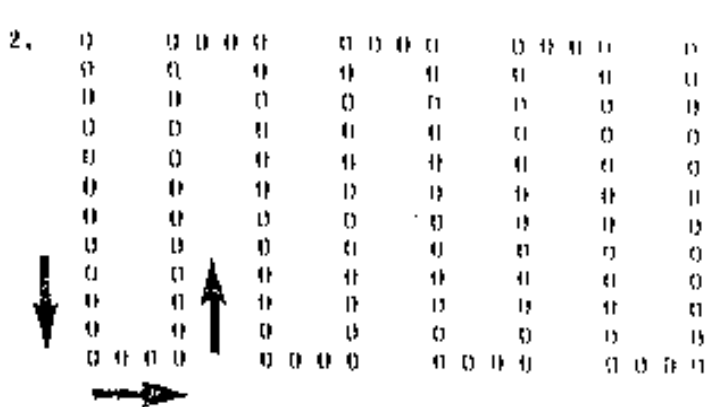

\section{Prefiered Hand}

\section{0 seconds per trial.}




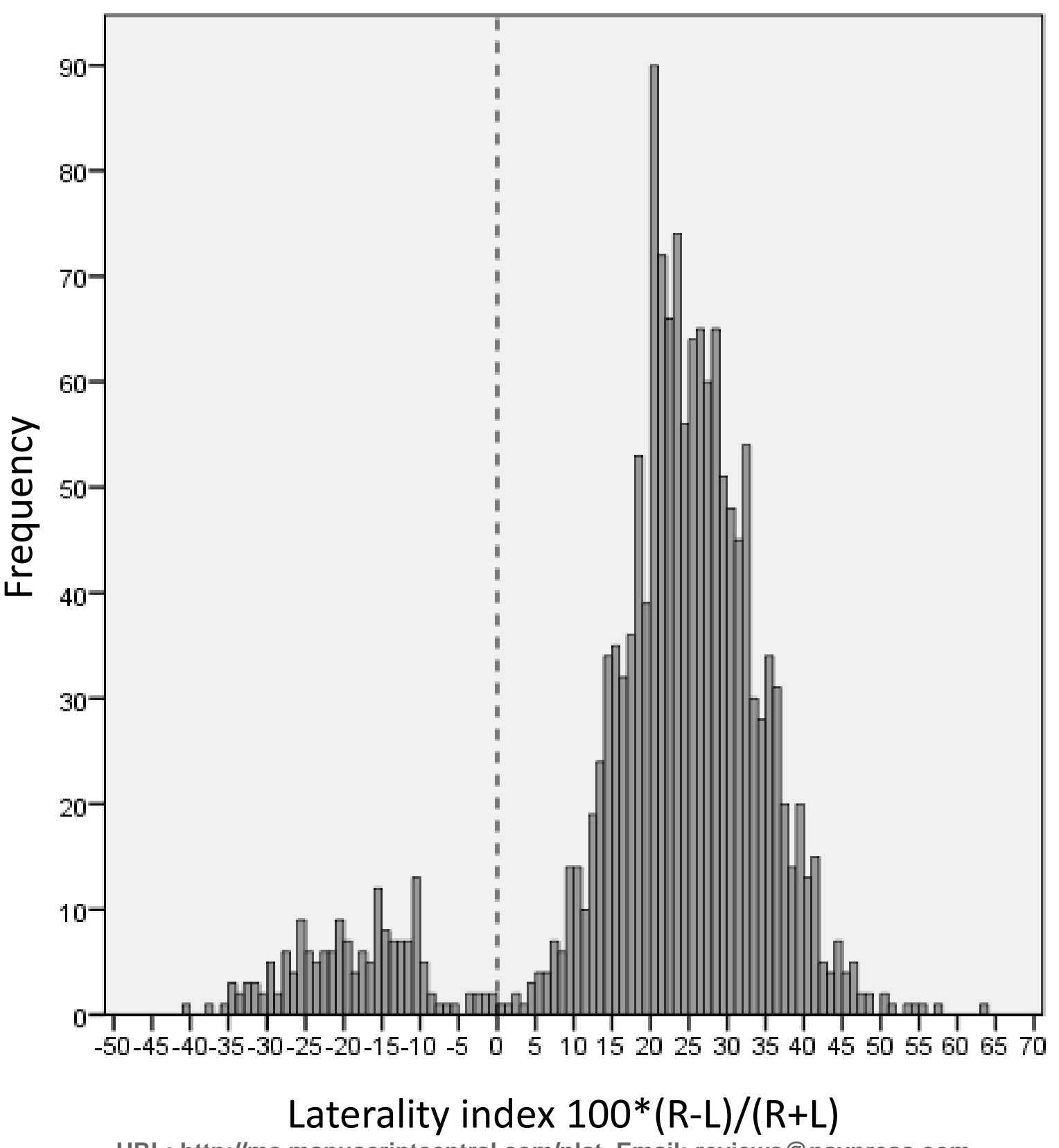




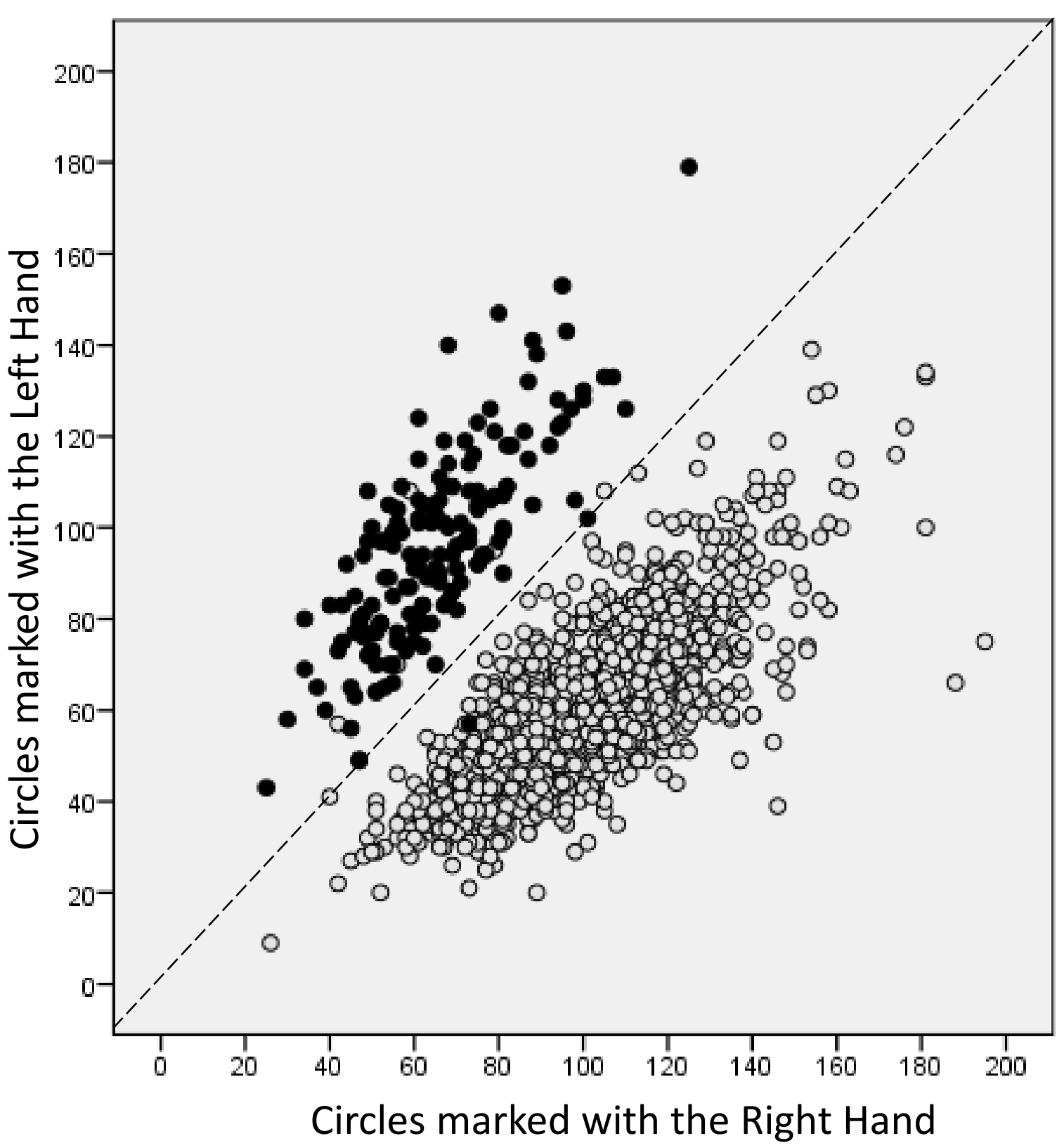




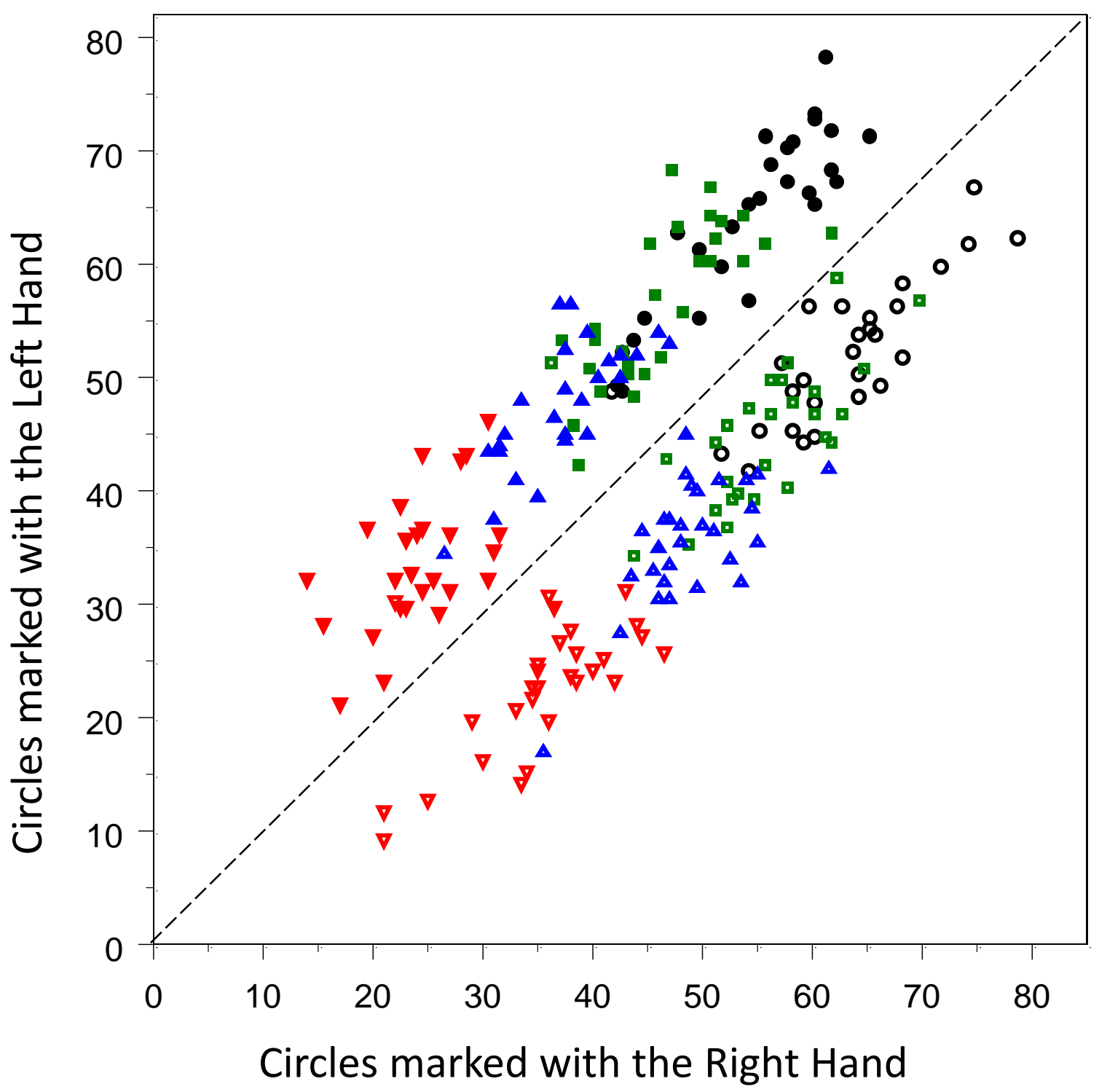




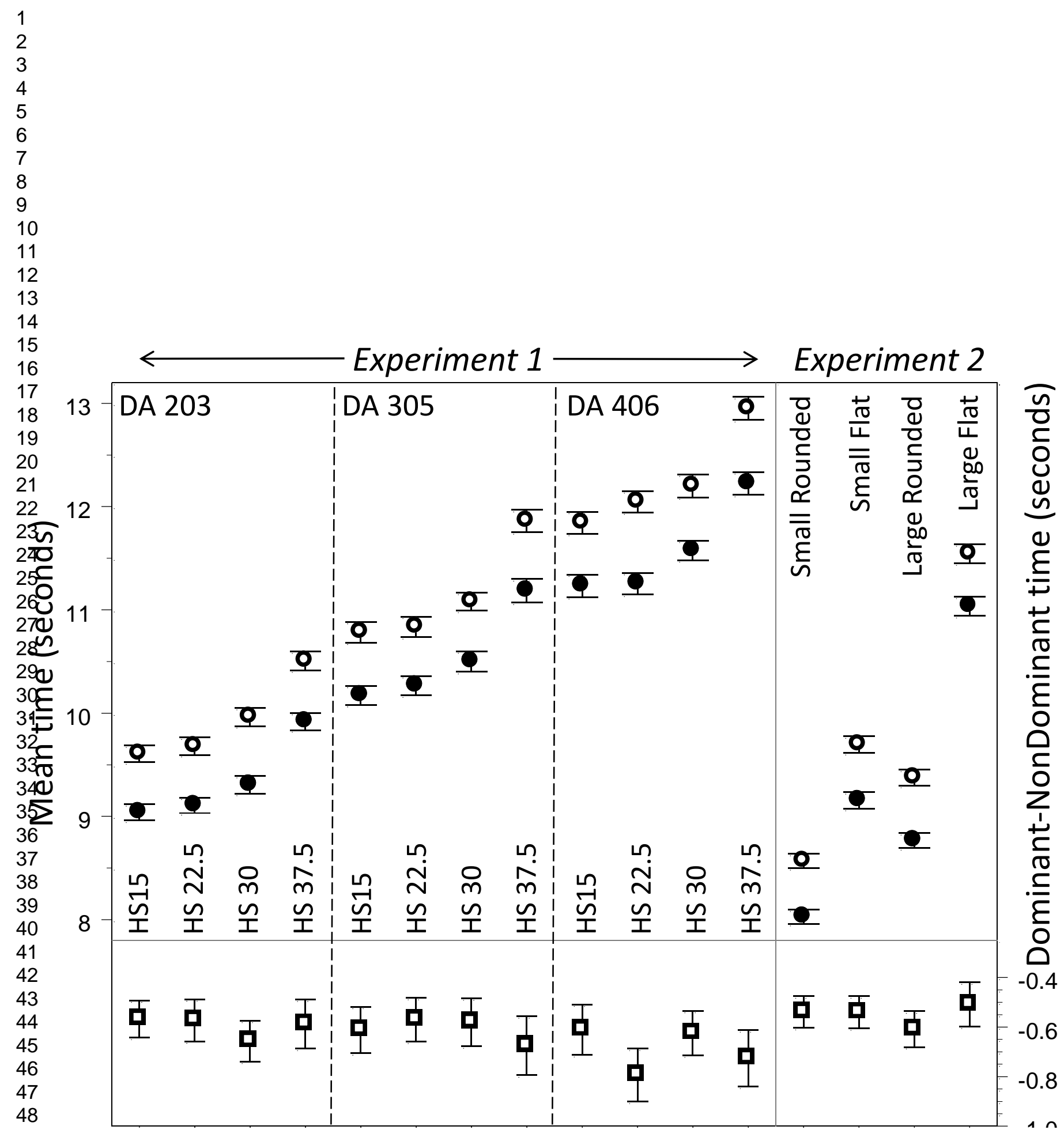




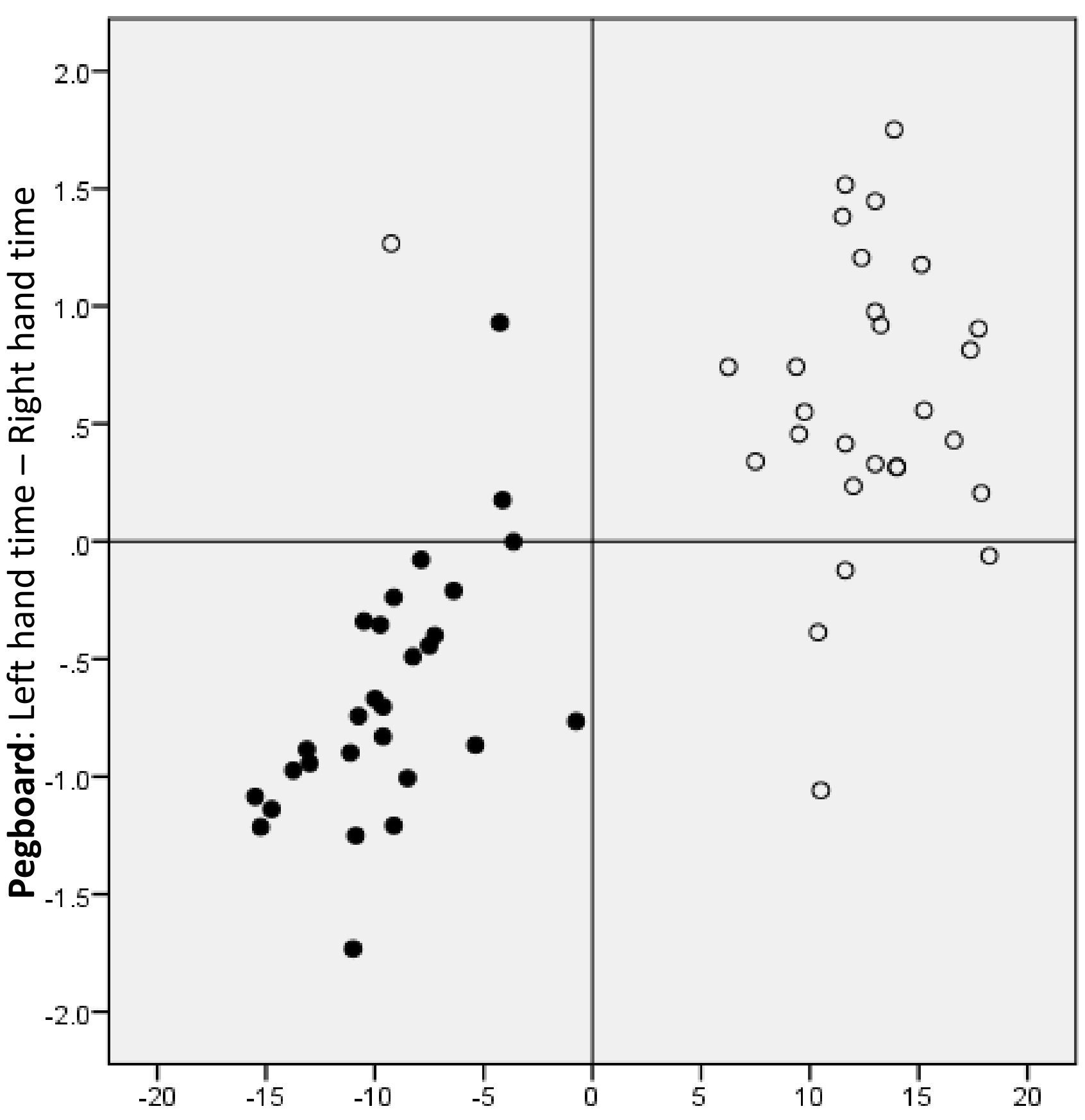



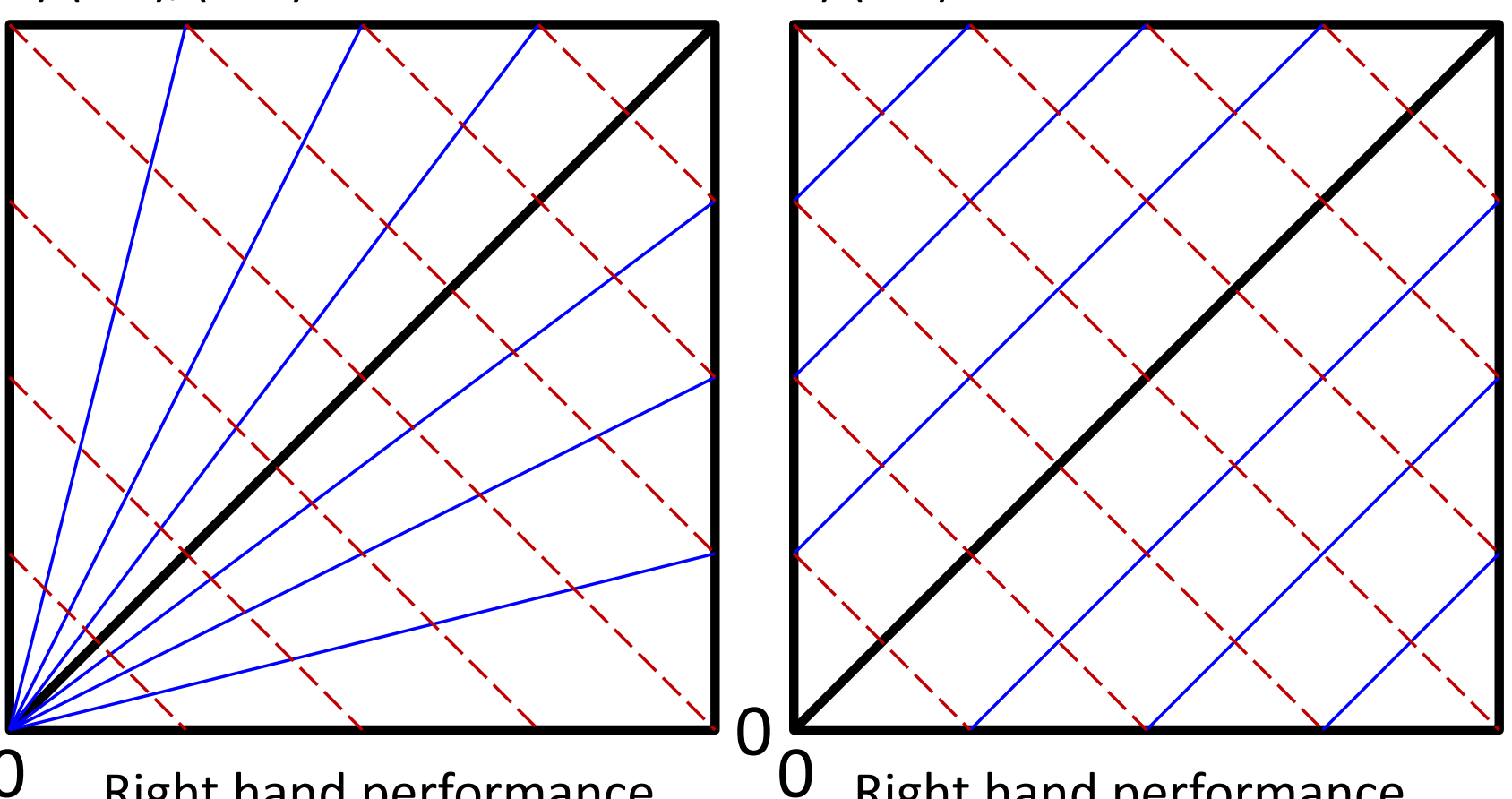

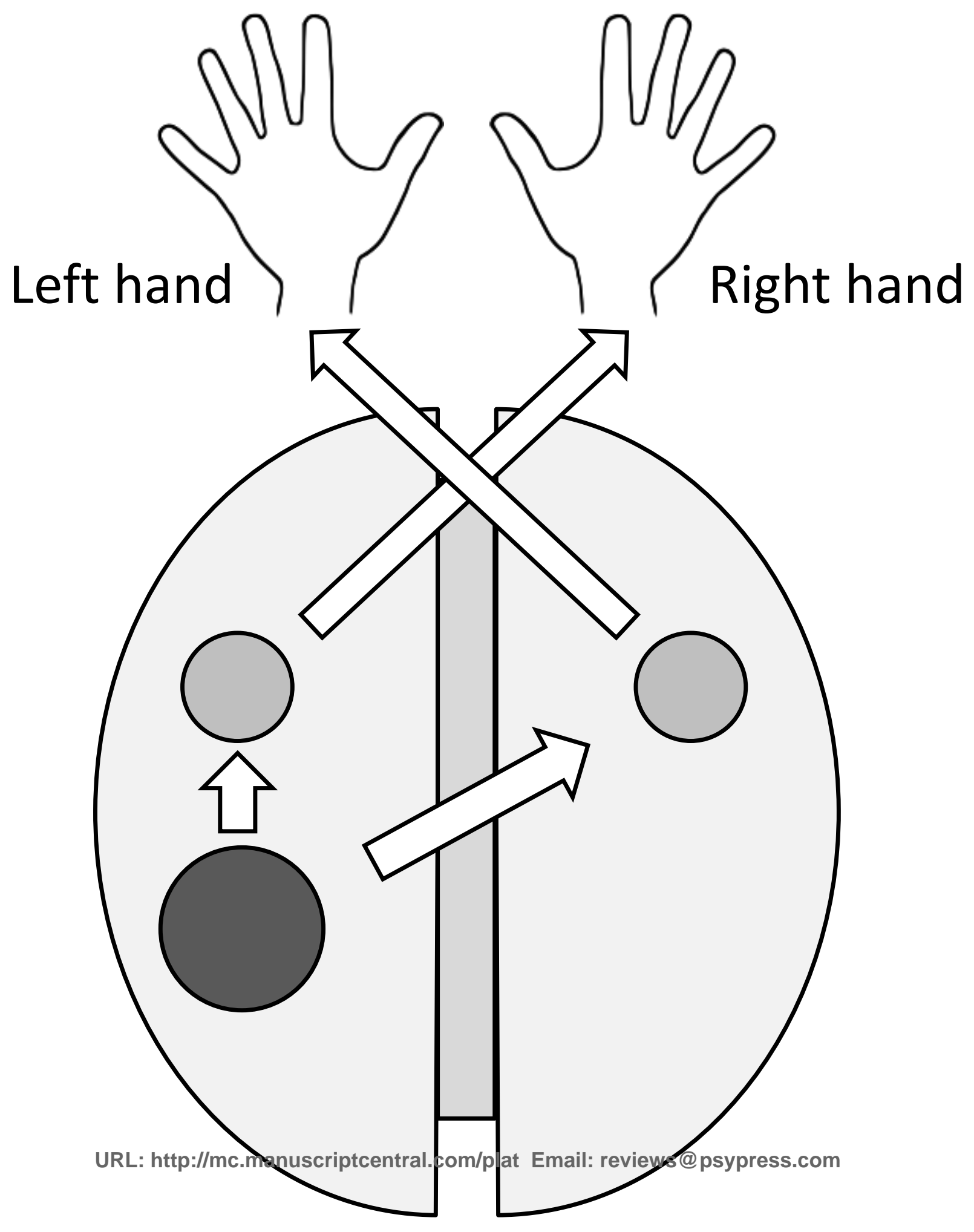